moderate length-dependent polyneuropathy of axonal type. Detailed blood screening studies were negative. Genetic testing revealed the diagnosis of nail-patella syndrome with LMX1B gene mutation on chromosome 9q34. The lack of an identifiable acquired cause and the symmetric, slowly progressive and "painless" nature of the patient's peripheral neuropathy point toward an inherited etiology. Conclusion: We present a case of slowly progressive sensorimotor axonal polyneuropathy in a patient with a diagnosis of NPS, which has not been previously reported. Peripheral nervous system disorder may be a variable phenotypic manifestation of LMX1B gene mutation.

\section{NeUROPHYSIOLOGY (FMRI)}

\section{P.063}

\section{Identification of resting state networks using independent} component analysis in patients with brain tumors

ST Lang (Calgary)* B Goodyear (Calgary) J Kelly (Calgary) P Federico (Calgary)

doi: 10.1017/cjn.2015.173

Background: Resting state functional MRI (rs-fMRI) provides many advantages to task-based fMRI in neurosurgical populations, foremost of which is the lack of the need to perform a task. Many networks can be identified by rs-fMRI in a single period of scanning. Despite the advantages, there is a paucity of literature on rs-fMRI in neurosurgical populations. Methods: Eight patients with tumours near areas traditionally considered as eloquent cortex participated in a five minute rs-fMRI scan. Resting-state fMRI data underwent Independent Component Analysis (ICA) using the Multivariate Exploratory Linear Optimized Decomposition into Independent Components (MELODIC) toolbox in FSL. Resting state networks (RSNs) were identified on a visual basis. Results: Several RSNs, including language $(\mathrm{N}=7)$, sensorimotor $(\mathrm{N}=7)$, visual $(\mathrm{N}=7)$, default mode network $(\mathrm{N}=8)$ and frontoparietal attentional control $(\mathrm{n}=7)$ networks were readily identifiable using ICA of rs-fMRI data. Conclusion: These pilot data suggest that ICA applied to rs-fMRI data can be used to identify motor and language networks in patients with brain tumours. We have also shown that RSNs associated with cognitive functioning, including the default mode network and the frontoparietal attentional control network can be identified in individual subjects with brain tumours. While preliminary, this suggests that rs-fMRI may be used pre-operatively to localize areas of cortex important for higher order cognitive functioning.

\section{NeUROSURGERY \\ (Critical Care/Neuro Trauma)}

\section{P.065 \\ "Novell" medical therapies in ICP management: targeting three brain states}

FA Zeiler (Winnipeg)* N Sader (Winnipeg) CJ Kazina (Winnipeg) J Teitelbaum (Montreal) LM Gillman (Winnipeg) M West (Winnipeg)

doi: 10.1017/cjn.2015.175

Background: There exists the role for novel agents in the management of refractory intracranial pressure (ICP) via targeting cerebral acidosis, hyperemia, and excitotoxicity. Objective: We performed 4 separate systematic reviews to determine the effect of tromethamine (THAM), indomethacin, and ketamine on ICP. Methods: All articles from MEDLINE, BIOSIS, EMBASE, Global Health, HealthStar, Scopus, Cochrane Library, the International Clinical Trials Registry Platform (inception to: February 2014 - THAM, July 2014 - Indomethacin, November 2013 - Ketamine), and gray literature were searched. The strength of evidence was adjudicated using both the Oxford and GRADE methodology. Results: Twelve articles were reviewed utilizing THAM while documenting ICP in neurosurgical patients. All but one study documented a decrease in ICP. Twelve original articles were reviewed utilizing indomethacin for ICP in neurological patients. All but one study documented a decrease in ICP. Seven articles were reviewed utilizing ketamine, documenting ICP in TBI patients, with 16 in non-trauma neurological patients. ICP did not increase in the studies during ketamine administration, and trended to decrease ICP. Conclusion: There exists Oxford level 2b, GRADE B evidence that THAM reduces ICP in the TBI and malignant ischemic infarct population. There exists Oxford level 2b, GRADE C evidence that indomethacin and ketamine reduce ICP in the adult severe TBI population.

\section{P.067}

The effectiveness of a concussion-u educational presentation on knowledge and attitudes of concussion amongst elite bantam and midget hockey players

M Eagles (St. John's)* M Powell (St. John's) D Bradbury-Squires (St. John's) J Murphy (St. John's) G Campbell (St. John's) FB Maroun (St. John's)

doi: 10.1017/cjn.2015.176

Background: The diagnosis of a concussion is often dependent on the athlete self-reporting their symptoms. It has been suggested that improving athlete's knowledge and attitudes towards concussions may increase self-reporting behavior; however, research in this area is inconclusive. The objective of this study is to determine if a Concussion-U educational presentation improves knowledge and attitudes of youth hockey players towards concussions. This is part of a larger study designed to determine the impact of an informational presentation on the knowledge and attitudes over a full hockey season. Methods: 56 elite male Bantam and Midget hockey players (mean age $=14.52 \pm 1.13$ years) were recruited from the local community. Each participant completed a modified version of Rosenbaum and Arnett's Concussion Knowledge and Attitudes Survey 
(RoCKAS) questionnaire immediately before and after a Concussion- $U$ educational presentation on the subject. Results: Concussion knowledge and attitude scores significantly $(\mathrm{p}<.001)$ increased from prepresentation to post-presentation by $13.1 \%$ and $8.7 \%$, respectively. Discussion: A Concussion-U educational presentation designed to improve concussion knowledge and attitudes in youth hockey players resulted in increased knowledge and improved attitudes towards concussion in elite male Bantam and Midget hockey players. Future research should examine the long-term effects of such presentations.

\section{P.068}

Epidemiological patterns of traumatic brain injury identified in the emergency department in Ontario, 20022010

\section{T Fu (Toronto)* R Jing (Toronto) M Cusimano (Toronto)}

doi: $10.1017 /$ cjn. 2015.177

Background: Traumatic brain injury (TBI) is the leading cause of traumatic death and disability, and most TBIs are treated in the Emergency Department (ED). We examined the incidence and epidemiological patterns of TBIs presenting to Ontario EDs over an eight-year period. Methods: All TBI-related ED visits between April 2002 and March 2010 were identified using a population-based database that is mandatory for ambulatory care facilities in Ontario. Incidence rates were reported across multiple strata, including age group, sex, and mechanism of injury. Results: From 2002-2010, there were 1,032,249 ED visits for TBI in Ontario. Peak rates occurred among young children ages 0-4 (349 per 10,000) and elderly adults ages $85+$ (243 per 10,000). Overall, males experienced a 53\% greater rate of TBI compared to females. Falls (47\%), motor vehicle crashes (MVC; $10 \%)$, and sports-related injuries $(9 \%)$ were the most common causes of TBI. The highest rates of TBI-related falls, MVCs, and sportsrelated injuries occurred among young children (0-4) and elderly adults (85+), adolescents/young adults (15-24), and children (5-14), respectively. Conclusions: Our study reveals a substantial health system burden associated with TBI in the ED setting, underscoring the need for enhanced surveillance and prevention efforts targeted to vulnerable demographic groups.

\section{P.069}

\section{Lifetime costs of traumatic brain injury identified in the emergency department in Ontario}

\section{$T$ Fu (Toronto) * R Jing (Toronto) M Cusimano (Toronto)}

doi: $10.1017 /$ cjn. 2015.178

Background: Traumatic brain injury (TBI) is a leading cause of death and disability, yet there is limited research on its economic burden. We estimated the incidence and lifetime costs of TBI identified in the Emergency Department (ED) in Ontario, Canada between April 2009 and March 2010. Methods: ED visits for TBI were identified using a population-based database that is mandatory for ambulatory care facilities in Ontario. The authors calculated unit costs for medical treatment and productivity loss, and multiplied these by incidence estimates to determine the lifetime costs of identified TBI cases. Results: In 2009, there were over 133,000 ED visits for TBI in Ontario, resulting in a conservative estimate of $\$ 945$ million in total lifetime costs. Costs were greater for males than females across nearly all age groups, with males incurring two-fold higher costs overall. Together, falls ( $\$ 407$ million), struck by/against (\$309 million), and motor vehicle injuries (\$161 million) represented $93 \%$ of lifetime costs associated with TBI. Conclusions: This study revealed a high incidence and economic burden associated with TBI identified in the ED. More research is needed to fully appreciate the burden of TBI across a variety of healthcare settings.

\section{P.070}

\section{Violence-related brain injuries sustained in youth ice hockey}

\author{
M Shirazi (Toronto) MD Cusimano (Toronto) * S McFaull (Ottawa)
}

doi: $10.1017 /$ cjn.2015.179

Background: Violence is a frequent occurrence in ice hockey and has been associated with an increased risk of injury. Methods: Case-control study. The study population consisted of youth aged 0-19 years who presented to a participating emergency department (ED) with an injury resulting from participation in ice hockey. In order to examine the outcomes following brain injuries in ice hockey, the outcome was admission to the hospital (cases) or discharge from the ED (controls). In order to examine the relationship between brain injury and violence, the outcome was brain injury (cases) or a nonbrain injury (controls). Logistic regression was used to determine the associations controlling for potential confounders. Results: In total, 56,835 youth suffered an injury related to ice hockey. $11.0 \%$ $(n=6,293)$ were brain injuries. Youth who engaged in violence were at significantly higher odds of sustaining a brain injury as opposed to an injury to another body part (OR: $1.67 ; 95 \%$ CI: 1.55-1.80). Youth who sustained violence-related injuries were at significantly higher odds of being admitted to the hospital compared to youth who sustained non-violence related injuries (OR: 2.34; 95\% CI: 1.49-5.68). Conclusions: Youth who engage in violence are at higher odds of sustaining a brain injury and of being admitted to the hospital.

\section{P.071}

\section{Brain injuries sustained by Canadian youth participating in Rugby Union}

M Shirazi (Toronto) MD Cusimano (Toronto)* J Di Michele (Toronto) S McFaull (Ottawa)

doi: $10.1017 /$ cjn.2015.180

Background: Rugby is a high-intensity, full-body contact sport in which there is an increased risk of injury associated with participation. The objectives of this study were to identify the mechanisms and characteristics of rugby-related injury sustained amongst Canadian youth. Methods: Data were obtained from the Canadian Hospitals Injury Reporting and Prevention Program database. The study population consisted of individuals aged 10-19 who sustained an injury while participating in rugby between the years 1990 and 2014. Proportions of body parts injured, mechanisms of brain injury, and nature of injury were calculated with $95 \%$ confidence intervals. Results: There were a total of 6200 rugby-related injuries sustained among individuals aged 10-19 between the years 1990 and 2014 . $16.0 \%(\mathrm{~N}=993)$ of all injuries were brain injuries, $48.9 \%(\mathrm{n}=486)$ of which were concussions. $70.7 \%(\mathrm{n}=4838)$ of all injuries were sustained by males. The predominant mechanism of brain injury was 